\title{
Une manufacture de céramique vue à travers ses déchets : expérience pluridisciplinaire autour d'un dépotoir
}

A ceramic factory read through its wastes: pluridisciplinary research around a dump

\section{Thierry Bonnot}

\section{OpenEdition}

Journals

\section{Édition électronique}

URL : http://journals.openedition.org/artefact/781

DOI : 10.4000/artefact.781

ISSN : 2606-9245

\section{Éditeur :}

Association Artefact. Techniques histoire et sciences humaines, Presses universitaires du Midi

\section{Édition imprimée}

Pagination : 11-28

ISBN : 978-2-7535-7305-5

ISSN : 2273-0753

Référence électronique

Thierry Bonnot, « Une manufacture de céramique vue à travers ses déchets : expérience

pluridisciplinaire autour d'un dépotoir », Artefact [En ligne], 6 | 2017, mis en ligne le 31 mai 2018, consulté le 06 mars 2020. URL : http://journals.openedition.org/artefact/781 ; DOI : https://doi.org/ 10.4000/artefact.781 


\section{Une manufacture de céramique vue à travers ses déchets : expérience pluridisciplinaire autour d'un dépotoir}

Thierry BONNOT*

\section{Résumé}

En France, l'archéologie industrielle s'est développée à partir des années 1970. Dans ce domaine, les opérations de fouilles demeurent assez rares, l'essentiel des recherches portant sur le bâti encore en élévation. Cet article retrace une expérience de fouille archéologique du contemporain, menée en Bourgogne, dans le dépotoir d'une entreprise de fabrication céramique daté $\mathrm{du} \mathrm{xx}^{\mathrm{e}}$ siècle, montrant que la problématique du déchet industriel est d'un intérêt considérable pour l'archéologie comme pour les autres sciences humaines, dont l'anthropologie.

Mots-clés : anthropologie, archéologie, céramique, déchets, industrie, patrimoine.

\section{Abstract. A ceramic factory read through its wastes : pluridisciplinary research around a dump}

Industrial archaeology in France expanded during the 1970s. In this fieldwork, excavations are quite unusual, most of the research focus on building still in elevation. This paper focuses on an archaeological excavation in Burgundy, in a $20^{\text {th }}$ century ceramic factory dump, showing that industrial wastes are significant for archeology as for other social sciences, including anthropology.

Keywords : anthropology, archaeology, ceramic, heritage, industry, wastes.

\footnotetext{
*. Thierry Bonnot est anthropologue, chargé de recherche au CNRS, membre de l'Institut de recherche interdisciplinaire sur les enjeux sociaux (IRIS, Paris). Ses recherches portent essentiellement sur le statut social des objets, leurs modes d'appropriation et la constitution des patrimoines, dans une optique pluridisciplinaire. Dernier ouvrage paru : L'attachement aux choses (Paris, éditions du CNRS, 2014). Contact : [bonnot@ehess.fr].
} 
C'est une découverte pas tout à fait fortuite, en 2011, qui est à l'origine de cet article. Jacques Gaudiau, collectionneur de céramiques rencontré à l'occasion de mes premières enquêtes dites " ethnohistoriques » dans la région du Creusot et de Montceau-les-Mines (Saône-etLoire), me contactait en juillet 2011 pour me faire part de quelques trouvailles intéressantes. Il avait identifié, près de chez lui, le lieu de déversement des déchets industriels de l'entreprise Paul Langeron, lieu connu par la tradition orale locale mais jusqu'alors non précisément situé. C'est le creusement d'un terrier de renard qui avait mis à jour les débris de poteries et de moules de plâtre permettant de fixer l'emplacement de la décharge. L'événement n'était pas totalement le fruit du hasard car nous savions que les déversements s'effectuaient sur cette parcelle, mais, grâce à la rencontre avec le propriétaire du terrain et grâce... au travail de Goupil, nous avions enfin accès à ce que les habitants du lieu-dit et les anciens ouvriers appelaient « les crasses » de l'usine Langeron.

Cette découverte posait à la fois un problème de compétence et de frontière disciplinaires. Il était évident que l'étude $\mathrm{du}$ site passerait par une démarche archéologique, donc par la sollicitation officielle d'une autorisation de sondage, bien que nous ayons affaire à de l'histoire contemporaine et à des objets potentiellement connus par les archives écrites et les collections des musées. Après avoir consulté des archéologues, le service régional de l'archéologie de Bourgogne, un spécialiste de l'histoire de la céramique ${ }^{1}$, il est apparu que Jacques Gaudiau et moi étions les mieux placés pour mener à bien cette opération bien que n'ayant aucune formation technique en archéologie. Quant à la frontière disciplinaire, cette expérience nouvelle en a montré la vacuité. La démarche anthropologique qui est la mienne n'est pas épistémologiquement incompatible avec l'engagement dans un tel projet, si l'on admet que l'enquête relève à la fois d'une science historique, d'un dialogue constructif avec nos interlocuteurs de terrain, d'une description circonstanciée des situations vécues « au ras du sol de la singularité », pour reprendre les termes d'Alban Bensa ${ }^{2}$. En l'occurrence, dans le cadre d'une recherche portant sur l'histoire d'une entreprise, de ses produits devenus objets de collection, de ses caractéristiques sociotechniques aussi bien que sur le processus de mise en patrimoine, les musées et les pratiques des collectionneurs, il s'avère indispensable d'utiliser tous les matériaux disponibles, aussi hétérogènes soient-ils, et de se confronter à toutes les modalités possibles de l'enquête en sciences humaines et sociales. La fouille archéologique en fait partie.

C'est la nature du terrain et la position même des objets dans l'espace, en l'occurrence sous terre, qui orienta l'entrée par l'archéologie, ne serait-ce que du point de vue juridique. De même, notre choix du mot « dépotoir » pour désigner ce que la mémoire orale nommait « les crasses ", situe notre démarche dans un cadre précis. Ce terme, concession à une terminologie spécialisée suggérée par un archéologue amateur, est tout sauf anodin. Il inscrit notre enquête, toutes proportions gardées, dans la lignée des recherches menées sur la production céramique par des générations d'archéologues sur les périodes antique, médié- 
vale et moderne. Étymologiquement, le terme " dépotoir » vient de " pot »; usuellement, il désigne le lieu où l'on verse les matières provenant des vidanges et, par extension, celui où $l^{\prime}$ on met les objets au rebut ${ }^{3}$. Les dépotoirs, qu'ils soient domestiques ou liés à la production, constituent une source majeure pour l'archéologie de la céramique et la spécialité céramologique ${ }^{4}$. En choisissant de transformer les « crasses » en dépotoir, nous prenions l'initiative de faire de ce terrain un objet de recherche et de transformer un lieu d'abandon de déchets en ressource archéologique et historique. L'objectif de cet article est de montrer comment la question des déchets constitue une passerelle pertinente et efficace entre ces disciplines.

\section{La céramique : une activité industrielle secondaire}

\section{Le bassin industriel et sa vallée de la céramique}

Le centre du département de Saôneet-Loire s'est industrialisé à partir des années 1830 autour de la métallurgie au Creusot et des houillères du bassin de Blanzy-Montceau-les-Mines ${ }^{5}$. Le long de l'axe transversal constitué par le canal du Centre joignant Saône et Loire, creusé à la fin du XVIII ${ }^{\mathrm{e}}$ siècle pour désenclaver ce département rural, une multitude d'entreprises ont profité de la proximité de ces deux industries majeures pour prospérer. La production céramique, assez éparse dans ce secteur en dehors de quelques centres anciens ${ }^{6}$ est l'un des secteurs d'activité qui a le plus bénéficié de ce contexte. Elle s'est principalement développée dans les vallées des rivières Dheune et Bourbince, le long du canal du Centre, des premières années du XIX ${ }^{e}$ siècle jusqu'aux environs de $1940^{7}$. L'apogée de cette "vallée de la céramique $^{8}$ » se situa au début du $x^{e}$ siècle, où une quarantaine de manufactures (tuileries, briqueteries, poteries) étaient en activité simultanément sur cette étroite bande de territoire, dont plus de la moitié dans le bassin industriel de Montceau-les-Mines et du Creusot. Après la Seconde Guerre mondiale, ce secteur d'activité a subi un déclin continu et ne subsistent aujourd'hui de cet ensemble que cinq entreprises à Digoin, Paray-leMonial, Palinges et Chagny?.

À peu près au centre de la vallée, à la lisière nord de l'arrondissement de Charolles, quelques manufactures se sont distinguées par leur production, le grès cérame, pratiquement inconnu dans cette région avant le $\mathrm{XIX}^{\mathrm{e}}$ siècle. Entre les années 1810 et 1960, un ensemble de petites (moins de dix ouvriers) et moyennes (au maximum une centaine d'ouvriers) entreprises se sont consacrées à la fabrication de poteries de grès fins glaçurés, destinées au conditionnement alimentaire et chimique. Les mêmes manufactures fabriquaient également des produits réfractaires, des briques de pavage, des tuiles de terre cuite ou de grès, et la dernière à fermer ses portes en 1976 produisait alors des faïences sani- 
taires après s'être essayée au funéraire. Mais l'homogénéité de cet ensemble résidait dans l'occupation d'un créneau de production et de commercialisation commun, l'utilisation de techniques similaires et la structure essentiellement familiale des entreprises. La fabrication de récipients pour conditionnement alimentaire, pharmaceutique, chimique ou parachimique, l'utilisation de matières premières (argiles et combustibles) locales, le façonnage manuel puis très partiellement et tardivement mécanisé des poteries, la couverture systématique des produits par une glaçure colorée ou non par des oxydes métalliques, la cuisson au charbon dans des fours à étages, la vente des produits finis à des industriels fournissant le contenu des récipients, pas ou très peu de vente directe aux particuliers : ainsi peut-on synthétiser les caractéristiques communes des poteries de grès du Charolais, en activité de 1811 pour la plus ancienne jusqu'au milieu des années 1970. L'apogée de cet ensemble industriel se situe entre 1860 et 1900, avec une douzaine d'entreprises actives simultanément, concentrées sur moins de vingt kilomètres le long de la Bourbince entre Pouilloux, au nord-est, et Palinges, au sud-ouest; plus loin vers l'ouest, des usines similaires étaient en activité à Paray-le-Monial (deux entreprises) et à Digoin (une entreprise). Les spécificités techniques, fonctionnelles et commerciales de ces entreprises reflètent la spectaculaire mutation des modes de vie dans les premières décennies de l'urbanisation et de l'industrie de masse en France : conditionnement alimentaire individualisé, démocratisation de produits de luxe, apparition de produits nouveaux.

\section{Une entreprise familiale durant 130 ans}

C'est au nord de ce chapelet d'usine que se trouve le lieu-dit le Pont-desVernes (commune de Pouilloux ${ }^{10}$ ). Ici, l'histoire de la fabrication céramique est indissociable du nom de la famille Langeron, à la tête de l'entreprise depuis sa fondation jusqu'à sa fermeture. Si le rapport du jury international de l'Exposition universelle de Paris, en 1900, date la fondation de la maison Langeron de $1800^{11}$, la première mention d'une fabrication de céramique à Pontdes-Vernes correspond à une tuilerie répertoriée par le cadastre napoléonien en 1815 sur la parcelle 381, l'une de celles où s'implantera l'usine de poteries ${ }^{12}$. Le plan en $L$ de la tuilerie correspond à celui d'un bâtiment que nous retrouvons sur les plans de l'usine Langeron jusqu'à sa fermeture. D'après les matrices cadastrales établies pour Pouilloux en 1823, le propriétaire de cette tuilerie est JeanPhilibert Langeron, éclusier à l'écluse du Four depuis 1810, après avoir occupé le même emploi à Ciry à la fin du XVIII $^{\mathrm{e}}$ siècle $^{13}$. Jean-Philibert Langeron est décédé en 1821, mais il est désigné comme propriétaire tant que la succession n'a pas été réglée, ce qui sera le cas en 1827. À cette date, l'ensemble de ses biens immobiliers à Pouilloux est partagé entre deux de ses enfants, Pierre et François. La tuilerie passe dans les mains de Pierre Langeron (1786-1843) qui est déjà connu comme tuilier en $1813^{14}$, sans certitude sur sa présence, alors, à la tête de la tuilerie de Pont-des-Vernes.

En 1836, Pierre Langeron est, selon l'état civil, "propriétaire ", avec un fils « fabricant » et un autre " potier $»^{15}$. 
Il s'agit de Jean et Adolphe-Pierre Langeron qui ont indemnisé leurs frères et sœur pour pouvoir continuer à exploiter l'entreprise ${ }^{16}$. Les deux frères Langeron se distinguent par le nom de leurs épouses : Jean Langeron-Brossard et Adolphe-Pierre Langeron-Baujard dirigent l'usine de Pont-des-Vernes ensemble après la mort de leur père en 1843, et se la partagent en deux lots en 1869. C'est à partir de cette date que nous distinguons l'usine dite « du bas » (au nord, Langeron-Brossard) et celle dite « du haut » (au sud, Langeron-Baujard). Jean Langeron-Brossard décède en 1885 et son fils Jules lui succède pour l'usine du bas. L'usine du haut prend davantage d'ampleur et Adolphe-Pierre LangeronBaujard la transmet en location à son fils Paul-Adolphe en 1877. Celui-ci décède en 1884, alors que son fils Octave n'a que 12 ans. La mère de ce dernier, Jeanne-Catherine, dite Jenny, fait appel à son neveu Paul-Eugène, conducteur de travaux à l'administration du canal $\mathrm{du}$ Centre, pour diriger l'entreprise. Les deux cousins forment verbalement, en $1895^{17}$, une société qui conserve la raison sociale " Paul-Langeron » héritée de Paul-Adolphe et identifiant l'usine jusqu'à sa fermeture. En 1902, l'usine du bas connaît d'importantes difficultés et Jules Langeron est déclaré en faillite $^{18}$. Les dirigeants de l'usine du haut rachètent celle $d u$ bas en empruntant à un banquier chalonnais ${ }^{19}$ et unifient l'ensemble, puis déposent, en 1904, une marque de fabrique portant la mention «PL-Pont des Vernes-France » inscrite dans un cercle ${ }^{20}$.

En 1919, Paul-Eugène et Octave Langeron établissent les statuts de la Société anonyme des établisse- ments Paul-Langeron, au capital de 350000 francs répartis entre huit actionnaires, tous membres de la famille Langeron. Les deux fondateurs sont administrateurs et directeurs et, lorsque Paul-Eugène meurt en 1922, c'est son fils Octave-Lazare qui lui succède. En 1937, c'est Octave (aîné) qui décède et son fils Paul-Adolphe qui prend sa place à la direction. Jusqu'à 1949, l'usine sera dirigée de facto par deux Langeron issus de deux branches familiales, et, de cette date à 1957, « Monsieur Paul » sera seul directeur après le départ d'Octave ${ }^{21}$. Entre-temps, la société anonyme était devenue, en 1941, société anonyme à responsabilité limitée dont les actionnaires étaient toujours des membres de la famille. Réunis en assemblée générale en novembre 1957, face aux difficultés commerciales récurrentes de l'entreprise, ils prononcent la dissolution anticipée et volontaire amiable, le projet de fermeture de l'usine étant affiché depuis juillet 1956.

Avant de commencer la fouille archéologique, nous disposions d'une bonne connaissance de l'entreprise, fruit d'enquêtes historiques et ethnologiques menées dans les années 1990-2000. Les collections d'objets, privées et muséales, étaient d'ores et déjà bien fournies et nous donnaient matériellement accès aux productions - mais uniquement aux productions commercialisées, donc aux objets conformes. Le processus technique était bien connu également par les témoignages des ouvriers et dirigeants, par la comparaison avec d'autres entreprises du même type - par exemple, Revol à Saint-Uze dans la Drôme -, par l'étude des manuels techniques des $\mathrm{XIX}^{\mathrm{e}}$ et $\mathrm{xx}^{\mathrm{e}}$ siècles. Toutefois, l'étude archéo- 
logique de ce dépotoir nous a montré combien nos connaissances restaient lacunaires dans le détail et comment une réflexion sur les ratés et les déchets du processus technique pouvait être pertinente pour l'histoire de l'industrie et de ses produits.

\section{La parcelle des " crasses"}

L'enquête menée à la fin des années 1990 auprès des habitants de la commune, des anciens ouvriers et des membres de la famille Langeron nous avait appris qu'il existait un important amoncèlement de débris de poteries, une "verse » ou des « crasses " pour reprendre la terminologie vernaculaire, sur un terrain de l'usine jouxtant d'anciens bâtiments en ruine. Après la fermeture de l'entreprise, en 1957, le site industriel avait été vendu en deux parties par les héritiers Langeron. La partie nord, du côté du pont sur le canal, avait été cédée à un particulier, avant même la cessation d'activité officielle de l'entreprise; la partie sud, le 18 juin 1962, à la Société anonyme des Confiseries de Bourgogne ${ }^{22}$, entreprise intéressée par les bâtiments industriels subsistants. Cette société fit construire de nouvelles structures en s'appuyant sur les anciennes, en laissa une partie à l'abandon (bâtiment des tours, fours et séchoirs datés de 1897) et se désintéressa des abords où la végétation commença à prospérer. L'entreprise, basée à Chalonsur-Saône, ne resta en activité à Pouilloux que quelques mois, jusqu'à la faillite prononcée le 6 novembre 1962, puis reprit son activité environ deux ans plus tard sous le nom des produits Sacébon-JeanLouis Liebaert et Compagnie, avant de fermer définitivement ${ }^{23}$. En 1966, le tri- bunal de grande instance de Mâcon, sur requête du syndic de faillite, prononça la vente des bâtiments et terrains. Mais il apparaît que l'entreprise de confiserie était encore propriétaire du terrain et des bâtiments en $1984^{24}$ et c'est un marchand de biens mâconnais qui servit d'intermédiaire pour le revendre, en 1985, à l'entreprise Aubœuf, actuelle propriétaire. Seuls les bâtiments récents intéressant cette dernière, la partie sud-ouest de la parcelle ne connut aucun aménagement et ne fut défrichée que partiellement et occasionnellement. C'est en 2011, grâce à Patrick Aubœuf devenu propriétaire après son père, que nous avons pu avoir accès à ce terrain.

La parcelle sur laquelle se situe le dépotoir objet de notre sondage, numérotée 386, section A (La Prat) sur le cadastre ancien, appartenait à la famille Langeron depuis 1839, ainsi que les parcelles adjacentes 385 et 387 . Toutefois ces parcelles ont été fragmentées et furent propriétés partielles d'autres habitants de Pouilloux jusqu'à ce qu'Octave Langeron achète l'ensemble en 1922. Nous ne pouvons malheureusement pas savoir si l'emprise du dépotoir faisait partie du terrain acheté à cette date, faute de précision des sources cadastrales et de l'acte de vente. Il est impossible par conséquent de savoir si le déversement a débuté en 1922 ou avant, même si, comme on le verra, la quasi-totalité des objets découverts peut être datée des années 1920 au plus tôt. 


\section{Les sondages archéologiques}

Deux campagnes de sondage ont été menées, chacune sur six mois (avrilnovembre) en 2012 et 2014. En dehors de l'évacuation de la couche supérieure de terre végétale et du creusement d'une tranchée par une pelle mécanique, l'ensemble de nos travaux a été effectué à la main (fig. 1, cahier couleur). Nous ne disposions que de peu de moyens humains - deux personnes, rarement trois - pour effectuer le terrassement et l'évacuation des déblais.

La première campagne (2012) a donné une vision d'ensemble de la zone de déversement et permis de collecter une importante quantité de mobilier archéologique (fig. 2, cahier couleur). La seconde série de sondages (2014) a tenu compte de la problématique ayant émergé deux ans plus tôt et a été menée de façon plus raisonnée, avec deux objectifs clairement définis : délimiter plus précisément la topographie et la stratigraphie des déversements; tenter d'affiner la chronologie du site.

\section{Topographie générale}

Nous ne disposons pas d'indices suffisants pour retracer les modalités de déversement à l'origine de l'utilisation du site : $y$ avait-il un terrain relativement plat sur lequel les dépôts ont formé un monticule? Les dépôts initiaux ont-ils servi à combler une carrière d'argile désaffectée, comme le cas est attesté sur d'autres sites? Nous avons pu toutefois en établir la topographie générale et les conditions d'utilisation. Le profil général du dépotoir est celui d'un tumulus au sommet aplani. C'est l'accumulation des débris qui a engendré la levée de terre jusqu'au plateau culminant. L'accès des véhicules chargés de déchets - des chars attelés, puis un camion à gazogène à partir des années 1940 - s'effectuait depuis la cour de l'usine et les principaux bâtiments de production par le nord, via un chemin longeant un bâtiment de tours et de séchoirs édifié en 1897, aujourd'hui encore en élévation mais menaçant ruine. Les véhicules gravissaient une pente ${ }^{25}$ qui s'accentuait au fil des années pour parvenir sur une plateforme constituée par les dépôts antérieurs et versaient leur chargement sur la pente opposée. Les tessons et autres débris dévalaient le dévers de façon plus ou moins accidentée selon leurs dimensions et leur forme : dans ce contexte, l'élément mobilier retrouvé au niveau le plus bas n'est pas forcément le plus anciennement déversé. Contrairement à un dépotoir résultant de l'accumulation successive de débris dans une fosse, qui permet la datation des dépôts par l'étude de la stratigraphie, puisque les éléments les plus anciens sont logiquement ceux qui se retrouvent au niveau le plus profond, cette décharge industrielle présente une configuration d'une lecture plus complexe. La première campagne a exploré cette pente de déversement et notamment la zone basse.

Grâce à une série de sondages ponctuels effectués au tractopelle de 5 mètres en 5 mètres, sur l'axe est-ouest d'accès des véhicules à la zone de déversement, ainsi que par l'étude topographique de l'axe nord-sud, nous avons pu estimer à mille ou onze cents mètres carrés environ $^{26}$ la surface totale du dépotoir. 
Nous avons constaté, après avoir atteint le sol naturel argilo-sablonneux, que l'épaisseur des dépôts était très irrégulière, de $270 \mathrm{~cm}$ à 370 , voire $400 \mathrm{~cm}$ de profondeur par rapport à la surface du sol actuel. Les arasements et tassements successifs ont aplani les niveaux supérieurs mais pas la base du dépotoir et il est vraisemblable que le sol d'origine était lui-même accidenté. Cette donnée topographique nous interdit d'évaluer le volume total des dépôts, à moins d'en rester à une valeur très approximative ${ }^{27}$.

\section{Recyclage, élimination, législation}

L'étude des déchets et rebuts abandonnés par l'entreprise sur le dépotoir nous a permis de distinguer trois types de déversements. D'abord, un déversement ordinaire lié au fonctionnement routinier de l'usine, qui concerne les déchets produits au quotidien : débris de défournement, ratés de cuisson ou d'émaillage isolés, déchets domestiques, moules usagés ou cassés. Ensuite, un ensemble de déversements exceptionnels, liés à des accidents de fabrication : ratés de cuisson ou d'émaillage regroupés dans une même zone, biscuits ou produits de même type regroupés dans une même zone, argile préparée mais non utilisée versée sous forme liquide. Enfin, une série de déversements liés à la démolition de certains fours et petits bâtiments et au nettoyage des ateliers après la fermeture de l'usine : lots de moules de plâtre (fig. 3, cahier couleur), matériaux de construction, lots de chaussures, lots de bouchons de canettes, etc.

À la sortie des fours, un tri des produits finis était effectué par les ouvriers afin de vérifier leur conformité aux exigences de la clientèle. En comparant les céramiques commercialisées - celles que l'on retrouve avec une étiquette du client, par exemple - avec celles mises au rebut, nous pouvons inventorier les défauts tolérés ou non : cloques, fissures, défauts de glaçures, uniformité de la couleur, etc. Ces constatations effectuées sur les objets eux-mêmes peuvent être recoupées avec des documents d'archives. Ainsi cette lettre de M. Lallier, représentant des établissements Langeron à Paris, en 1872 :

"Si vous avez des cruchons d'un litre au sel et fait dans les contenances de 98 à 102, mais bien régulier, vous pouvez m'en expédier 2 cadres, vous savez qu'il ne m'en faut pas au-dessus ni au-dessous. Monsieur Antoine s'est plaint de votre bouteille qui est "bavarde ${ }^{28 "}$, faites donc bien attention, je crois qu'elle n'est pas assez cuite ou pas assez émaillée à l'intérieur ${ }^{29}$. »

C'est bien l'utilisateur du produit qui impose ses normes au fabricant et influe sur le processus technique, quitte à accroître la quantité de produits non conformes, donc de rebuts. Une partie de ces derniers pouvait être recyclée : si elles ne relevaient ni du premier ni du second choix, les céramiques non commercialisables pouvaient être utilisées comme récipients pour les besoins de l'entreprise si leurs défauts le permettaient. Le reste, ce qui était trop défectueux, pouvait être utilisé comme remblai, par exemple pour combler les carrières d'argiles désaffectées, pour aménager un gué dans le lit $\mathrm{d}^{\prime}$ une rivière ${ }^{30}$ ou pour être revendu à d'autres entreprises. Les produits réfrac- 
taires usagés pouvaient aussi être broyés afin d'être intégrés à la chamotte et revenir dans le cycle de fabrication. Les moules de plâtre, les oxydes métalliques et produits chimiques destinés à la fabrication des glaçures, les débris métalliques ou certains objets potentiellement polluants (piles électriques, batteries) ne pouvaient pas être remployés par l'entreprise; ils étaient jetés sans discernement sur le dépotoir, en très faible quantité cependant. La prise de conscience des risques environnementaux liés à de tels rejets est intervenue postérieurement à la fermeture de l'entreprise.

L'élimination de déchets industriels en pleine nature, à proximité immédiate d'un ruisseau et des habitations, comme $c^{\prime}$ est le cas du dépotoir de Pont-desVernes, n'a rien d'exceptionnel au début $\mathrm{du} \mathrm{xx}^{\mathrm{e}}$ siècle étant donnée la législation en vigueur. Concernant l'industrie, la législation mise en place à partir du début du XIX ${ }^{\mathrm{e}}$ siècle était principalement destinée à l'espace urbain, plus particulièrement à l'agglomération parisienne, et visait d'abord à éviter les nuisances olfactives ou les émanations chimiques dangereuses $^{31}$. Cette prise de conscience des problèmes posés par les usines ne concernait que l'activité en elle-même et les nuisances produites par le processus de fabrication (fumées et odeurs). Le problème des déchets et de leur élimination ne faisait l'objet d'aucune législation spécifique, hormis pour certains produits chimiques à ne pas déposer à même le sol. Si le fonctionnement de l'industrie et ses conséquences sur le voisinage constituent pendant la révolution industrielle un enjeu politique et économique impor$\tan \mathrm{t}^{32}$, la thématique environnementale n'apparaît pas encore sous ce vocable.
Pour ce qui est des usines travaillant des matériaux a priori non dangereux, la réglementation de l'élimination et du recyclage des déchets laisse toute latitude aux industriels, tant qu'ils entassent leurs déchets sur des terrains leur appartenant. La législation demeure presque muette jusqu'aux années 1970 en France où les décharges brutes, non contrôlées, sont largement tolérées. Il faut attendre une loi de 1975 pour que soit véritablement régulées l'élimination des déchets et la récupération des matériaux ${ }^{33}$.

\section{Le mobilier archéologique et sa datation}

C'est la fouille de la couche supérieure $\mathrm{du}$ dépotoir, après décapage de la terre végétale couvrant l'ensemble, qui s'est avérée la plus fructueuse pour la collecte de mobilier archéologique ${ }^{34}$. Elle a donné accès aux déchets les plus récemment évacués, correspondant à la fin d'activité et au nettoyage des ateliers de 1956 et 1957. L'essentiel de ces dépôts concernait des accessoires encore en bon état, parvenus jusqu'à nous assez bien conservés. C'est vrai en particulier pour les moules et modèles de plâtre que la fouille archéologique nous a permis littéralement d'inventer : nous connaissions ces éléments du processus de fabrication par les archives et les témoignages, mais leur existence demeurait largement virtuelle car nous n'étions parvenus à en retrouver et à en conserver que de rares exemplaires. Grâce à la fouille, l'écomusée Creusot-Montceau dispose désormais d'une importante collection de moules de coulage, calibrage et estampage ${ }^{35}$, mais aussi de modèles et de matrices ou moules-mères, le tout destiné à la pro- 
duction en grande série d'objets parfois non encore répertoriés. D'autres éléments essentiels de compréhension du fonctionnement de l'usine sont issus de ces déversements de fin d'activité. Ainsi les éprouvettes, objets céramiques sur lesquels étaient réalisés des essais de glaçure ou de marquage, voire de mélanges de matières premières. Des nuanciers de couleurs ont été également collectés, portant mention de la densité de la glaçure et des proportions d'oxyde métallique correspondant à la couleur de la poterie. Des essais de fusion de matières premières, réalisés dans des couvercles céramiques, nous donnent également accès aux tâtonnements des céramistes et à un certain empirisme du processus de fabrication industrielle dans un domaine assez pauvre en matière $d$ 'innovation technologique. Enfin, des outils ou des accessoires faisaient partie de ces déchets issus du nettoyage des ateliers : baleines de tour à potier, cent soixante-douze estèques, supports réfractaires, tampons pour marquage, étiquettes céramiques pour casiers, etc.

À quelques rares exceptions près, trop isolées pour être significatives, les indices de datation fournis par les objets collectés forment un faisceau concordant qui nous renvoie à la période postérieure à 1920. C'est une période déterminante pour l'évolution technique de l'entreprise. En mai 1919, le conseil d'administration de la société anonyme des établissements Paul Langeron décide $\mathrm{d}^{\prime}$ installer un atelier de coulage à la suite de la demande de "plusieurs clients ${ }^{36}$ ». En décembre 1920, sont soumises à l'approbation du conseil d'administration " les différentes adjonctions apportées à la marche de l'usine telles que l'installa- tion d'un atelier de coulage et d'un atelier de fabrication mécanique ». Le rapport précise que ces ateliers fonctionnent déjà « depuis plusieurs mois et donnent de très heureux résultats à tout point de vue », encourageant l'entreprise à persévérer $^{37}$. Les industriels, tout en maintenant la fabrication manuelle et le recours aux tourneurs-potiers, s'engagent dans une mécanisation plus poussée et une proportion importante de poteries sont produites par moulage (calibrage ou coulage). Ces produits moulés se retrouvent à tous les niveaux du dépotoir, de la surface au fond de la tranchée sondage de 2014. Même les déversements les plus anciens, en profondeur, ont donc été effectués après 1920.

\section{Mutation technique et gestion des déchets industriels}

Les traces matérielles laissées par l'activité des industriels montrent que l'évacuation des déchets après cette date a fait l'objet d'une réflexion et d'aménagements. À l'est et au sud-est, la parcelle est délimitée par un ruisseau qui débouche dans le canal du Centre. Le défrichage et le dégagement du léger talus formant un décrochement au-dessus du ruisseau ont permis de constater qu'il est formé de pierres de construction (grès et calcaire), de briques (terre cuite et réfractaire) et de plaques ou fragments de plaques réfractaires (débris de four), non maçonnées mais régulièrement empilées. Il s'agit d'un alignement de matériaux de récupération formant muret, prolongé vers le sud-ouest par un empilage continu quoiqu'irrégulier, parallèle au ruisseau, constitué de pierres plus imposantes, que nous n'avons pas pu intégralement 
dégager faute de temps. À son extrémité nord, le muret s'élève à une hauteur maximum de 80 centimètres, sur trois rangées verticalement (fig. 1). Il s'agit d'un aménagement du site du dépotoir marquant la limite avec la parcelle voisine et évitant le déversement massif de débris dans le ruisseau contigu. Le muret s'interrompt au nord pour laisser place à un épandage de matériaux de même nature (pierres et matériaux de construction). Après défrichage, une zone relativement plane d'une dizaine de mètres carrés se dégage, compactée par le passage régulier de véhicules. C'est sur cette plateforme, s'ouvrant d'un côté sur le chemin longeant le bâtiment de 1897, de l'autre sur la pente du dépotoir, que manœuvraient les véhicules chargés de

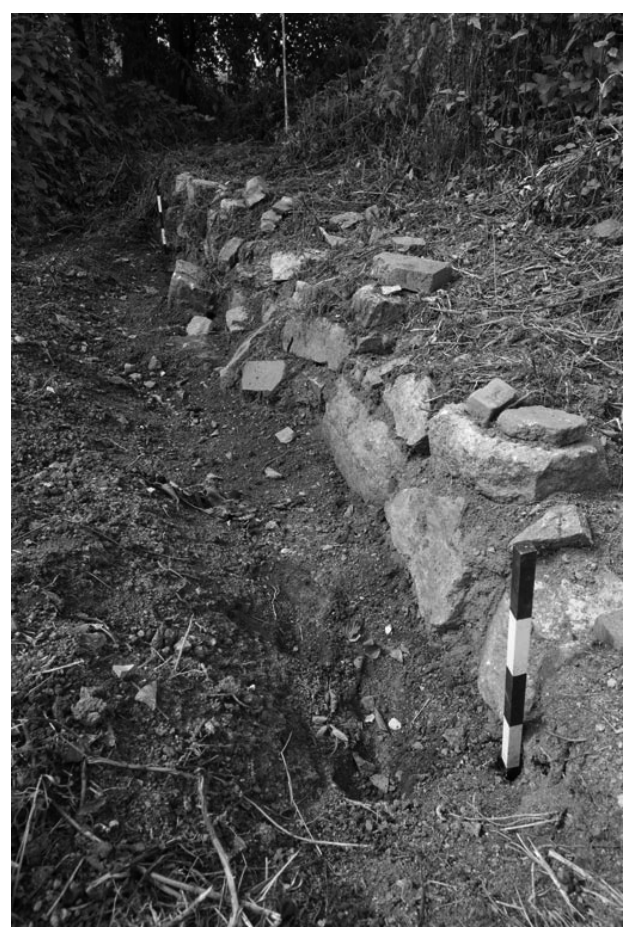

Figure 1. - Muret de matériaux non maçonnés bordant la parcelle, vue du nord-est, sondage 2014. Cliché de l'auteur. débris afin de gravir la pente en marche arrière. Cet aménagement, combiné à la construction d'un muret de clôture marquant clairement un bornage du site, fait partie d'une politique de rationalisation $\mathrm{du}$ traitement des déchets industriels par l'entreprise Langeron. Au-delà de la simple décharge constituée d'entassement désorganisé de déchets, la façon humaine la plus ancienne et la plus commune de résoudre la question ${ }^{38}$, il y a là une ébauche de réflexion pratique sur un problème exponentiel.

Dans la même logique, le sondage de 2014 a fourni des indices stratigraphiques probants. L'ouverture d'une tranchée ${ }^{39}$ parallèlement à l'orientation des déversements, au cœur de l'accumulation la plus massive des dépôts, nous a permis de travailler par niveaux. Sous la couche de surface de terre végétale, $\mathrm{d}^{\prime}$ une épaisseur irrégulière ${ }^{40}$, se trouve une couche d'environ $60 \mathrm{~cm}$ d'épaisseur composée de démolition de bâti, gravats, fragments de tuiles mécaniques, matériaux réfractaires broyés, terre argilo-cendreuse. Viennent ensuite : une troisième strate d'une épaisseur de $30 \mathrm{~cm}$ composée de sable réfractaire et de briques réfractaires broyées; un niveau très dense de tessons de grès cérame, d'une épaisseur de 60 à $80 \mathrm{~cm}$; sous ces quatre couches se trouvent les déchets de production (ratés de cuisson ou d'émaillage, invendus ${ }^{41}$ ) (fig. 4, cahier couleur). Certains objets sont quasiment intacts, comme s'ils avaient été déposés plutôt que jetés. La fouille permet de distinguer très nettement ces produits céramiques éliminés pour un défaut d'émaillage ou une déformation, des tessons, poteries fragmentées plus ou moins grossièrement, délibérément, comme pour trans- 
former des récipients en matériau de remblai.

L'horizontalité des couches dans la partie centrale de la zone atteste de leur arasement, voire de leur tassement par des moyens mécaniques, sans doute par un véhicule lourd, arasement destiné à maintenir une surface plane accessible aux véhicules de transport des déchets. L'homogénéité des matériaux de chaque couche suppose que les différents types de déchets n'étaient pas mélangés avant déversement sur le dépotoir. Les cendres, les débris réfractaires, les poteries et autres gravats étaient entassés séparément, dans la cour de l'usine, par exemple. Cette succession de couches homogènes, constituée progressivement durant l'occupation du site, résulte du fonctionnement régulier de l'usine, d'un mode d'élimination des déchets sur la pente des déversements mise en évidence par le sondage de 2012, à l'ouest de la zone. Cette variation topographique correspond à deux périodes de fonctionnement du dépotoir, celle de l'activité industrielle et celle suivant la fermeture de l'usine. Ces derniers dépôts n'ont pas été aplanis, mais l'ensemble a été alors couvert de terre végétale.

Ces différentes opérations - tri des matériaux avant déversement, arasement, compactage - visent à une stabilisation des sols pour un usage plus efficace du site, notamment par l'amélioration de la sécurité et la diminution de la surface d'épandage. Ces pratiques sont à rapprocher des aménagements des abords et du bornage signalés précédemment, attestant d'une réelle démarche d'optimisation du travail industriel, prenant pleinement en compte l'évacuation des rebuts et déchets de production.

\section{La question du déchet entre histoire, archéologie et anthropologie}

Notre travail s'inscrit dans la lignée d'une archéologie industrielle qui s'est développée en France dans les années 1970, inspirée par le modèle anglais, sur un terrain pionnier dans ce domaine, à savoir le bassin industriel du Creusot et Montceau-les-Mines.

Il convient toutefois de préciser ce que recouvre la notion d'archéologie industrielle et ce qu'elle implique d'un point de vue méthodologique et épistémologique. Comme le soulignent Florence Journot et Gilles Bellan, le point de vue des chercheurs de ce champ émergent
" était novateur à plus d'un titre : non seulement en qualifiant d'archéologiques des structures et du mobilier datant $\mathrm{du} \mathrm{XVII}^{\mathrm{e}}$ au $\mathrm{xx}^{\mathrm{e}}$ siècle, mais encore parce que la fouille de ces installations n'était pas obligatoirement envisagée, les "sites" pris en compte n'étant pas enfouis, qui plus est en élévation et souvent encore en usage ${ }^{42}$ ».

Il s'agissait principalement d'inventorier et de décrire les bâtiments subsistants de l'activité industrielle dans un 
but militant de sauvegarde, de conservation et de mise en valeur et, à cet égard, l'ouvrage considéré comme fondateur signé en 1980 par Maurice Daumas ${ }^{43}$ a pu laisser certains sur leur faim. Ainsi en fut-il de Jean-Claude Beaune, philosophe des techniques, déplorant dans un compte rendu de ce livre, pourtant attendu " avec curiosité et impatience ", qu'il ne s'agisse que " d'un répertoire, d'un travail résultant d'une accumulation de fiches ", n'apportant rien quant aux « méthodes, [aux] enjeux philosophiques, scientifiques surtout de l'archéologie industrielle ${ }^{44} »$. J.-C. Beaune voit dans cette absence d'ambition synthétique et prospective un signe de la faiblesse de l'histoire des techniques en France, sans s'attarder sur le point qui nous retiendra ici : pourquoi archéologie et non histoire industrielle ou mieux, patrimoine industriel? Car c'est cette dernière formule qui l'a peu à peu emporté, même si la revue de référence de la spécialité a conservé son titre $\mathrm{d}^{\prime}$ origine ${ }^{45}$ en se consacrant essentiellement à la mémoire industrielle, matérialisée dans le bâti, les paysages et les machines, comme l'indique explicitement son soustitre : Patrimoine-technique-mémoire.

Autour du Creusot, si le travail de recherche et de valorisation du patrimoine industriel fut considérable et demeure emblématique, les fouilles à proprement parler y furent très rares et n'ont laissé que peu de traces dans les archives de l'écomusée local ${ }^{46}$. F. Journot et $\mathrm{G}$. Bellan signalent toutefois quelques expériences très intéressantes, notamment celle menée sur le site de la manufacture Gréber dans le Beauvaisis ${ }^{47}$, mais il est certain que ce champ de recherche ne donne lieu qu'assez rarement à des fouilles archéologiques telles qu'on les conçoit généralement. La question de la fouille ne constituait d'ailleurs pas un enjeu majeur pour les chercheurs des années 1970-1980, la question première étant bien de s'intéresser aux éléments matériels renvoyant à l'activité industrielle des hommes ${ }^{48}$, jusqu'alors négligée, voire méprisée. Pour Philippe Boissinot, l'archéologie industrielle " s'attache principalement à la valorisation du patrimoine industriel à un moment où celui-ci est manifestement menacé mais souvent encore en élévation $^{49}$ », ne nécessitant donc pas d'opération de fouille. Désigner cette démarche comme une archéologie relèverait donc selon lui d'une volonté d'établir un lien dans la longue durée - du Paléolithique à nos jours - entre artefacts et lieux de production, afin de "donner l'illusion que l'on s'intéresse au même projet humain, selon des méthodes similaires ${ }^{50} »$. Dans son ambition de définir rigoureusement les contours de sa discipline, P. Boissinot se montre ainsi réservé, pour ne pas dire sceptique, sur ce qui relève d'un usage métaphorique du mot archéologie ${ }^{51}$, ainsi que sur certaines démarches iconoclastes concernant les périodes récentes dont les apports tiennent plutôt de l'anecdote ou même du trucage ${ }^{52}$. À cet égard, l'enfouissement, nécessitant la fouille, peut être vu comme l'une des conditions de légitimation de l'archéologie du contemporain en ce qu'il implique « la mise hors circuit des choses, qui ne sont dès lors plus engluées dans une continuité, et que l'on amène à surgir dans un monde qui n'est plus le leur ${ }^{53}$ ».

$\mathrm{Du}$ moment que son étude nécessite de fouiller le sol, la matérialité des périodes récentes peut donc légitime- 
ment être l'objet d'une archéologie. C'est aussi ce que disent, mais comme en miroir car d'un point de vue nettement distinct du précédent, les archéologues Philippe Bruneau et Pierre-Yves Balut pour défendre l'extension chronologique du champ de l'archéologie. Ils appellent à " récuser la quasi équivalence largement accréditée aujourd'hui et amplement développée par les professionnels, de l'archéologie et de la fouille ${ }^{54} »$. Cette " identification de l'archéologie tout entière à la fouille » obligerait en effet à " définir l'archéologie par les conditions extra-scientifiques de la recherche ", comme si l'on définissait les sciences de la nature selon qu'elles utilisent le microscope ou " l'expérimentation en milieu aseptique ${ }^{55} »$. D'autre part, et les auteurs en font la seconde base de leur tentative de déplacer les frontières de la discipline, cette assimilation archéologie/fouille pose le problème de la définition de l'objet propre à l'archéologie qui pourrait dès lors correspondre à cet énoncé réducteur : " ce qui n'est pas enterré ne paraît pas archéologique [et] les frontières de la science sont celles du trou ${ }^{56} "$, alors que nous savons que l'enfouissement d'un objet peut être fortuit donc en aucun cas constituer un argument scientifique. Ce point critique nous intéresse directement sur notre terrain où la fouille du dépotoir nous a confrontés à des types d'objets déjà présents dans les collections publiques ou privées. Il n'y a évidemment aucune différence de nature entre la poterie de grès découverte dans une cave, un grenier ou sur l'étal d'un brocanteur et la poterie du même modèle extraite d'un agglomérat de cendres, d'humus et d'argile. Nous ne pouvons pas, en toute logique scien- tifique, qualifier celle-ci d'archéologique pour la distinguer de celle-là qui serait historique, patrimoniale ou muséale. Nous rejoignons alors l'idée de Laurent Olivier pour qui les vestiges archéologiques deviennent par la fouille des objets du présent qui «sont désormais ici, avec nous qui les déchiffrons et tentons d'établir les histoires dont ils procèdent ${ }^{57}$ ». Le travail sur le présent, sur les situations observées dont les objets font partie ainsi que les différents acteurs impliqués dans l'opération de recherche, est un travail anthropologique : pour l'anthropologue, pas de rupture radicale entre ce qui se passe ici et ce qui s'y est passés8.

Les enjeux de notre expérience archéologique et anthropologique ne se situent donc ni au niveau de la fouille comme critère fondamental d'archéologicité ${ }^{59}$, ni sur le plan chronologique de la légitimité d'une archéologie du récent. Celle-ci est désormais pleinement admise si l'on en croit la nouvelle programmation nationale de la recherche archéologique présentée en 2016 par le CNRA ${ }^{60}$. Notre travail se veut davantage une réflexion sur les rapports entre la production industrielle des $\mathrm{XIX}^{\mathrm{e}}$ et $\mathrm{xx}^{\mathrm{e}}$ siècles, telle qu'elle nous apparaît par ses produits finis et commercialisés - ceux qui se trouvent aujourd'hui intégrés aux collections -, et les processus techniques, ses tâtonnements, ses ratés même auxquels nous a donné accès la fouille du dépotoir. L'adoption d'un angle d'analyse qui se démarque de la tradition patrimoniale en exploitant les déchets, matériau inaccessible ou négligé jusqu'alors, constituait le pari initial de la démarche. La pertinence de l'archéologie du déchet a été démontrée dans d'autres cadres et nous l'expérimentons au niveau de 
la petite industrie. L'étude des déchets et rebuts s'avère très heuristique en ce qu'elle donne accès de façon fine au processus de fabrication, au quotidien de la production, aux échecs techniques et commerciaux des industriels, aux tactiques empiriques et routinières d'adaptation aux contraintes de la matière. Le travail du musée, comme celui du collectionneur privé, respecte communément une logique de catalogue. L'objet de collection idéal et inventorié doit être intact, le plus complet possible, avec l'étiquette $\mathrm{du}$ client ou son bouchon céramique d'origine le cas échéant, voire avec son contenu $^{61}$. L'enquête anthropologique analyse précisément ces pratiques en les inscrivant dans le cadre plus large du rapport social aux objets et au patrimoine $^{62}$. La découverte du dépotoir et son étude nous ont amené à nous intéresser au tesson, à la malfaçon, au raté de cuisson, ce qui relève d'une toute autre démarche et implique un engagement physique, un rapport frontal à la matière et aux éléments. Notre enquête a dû composer avec le paradigme cynégétique de Carlo Ginzburg. Nous avons accompli " le geste peut-être le plus ancien de l'histoire intellectuelle du genre humain : celui du chasseur accroupi dans la boue qui scrute les traces de sa proie $^{63}$ ». Accroupis au fond de la tranchée de sondage, en tentant de juguler les éboulis des parois, nous avons repéré et collecté les tessons et débris abandonnés par les céramistes, traces matérielles d'un processus de fabrication dont les archives écrites ne nous sont parvenues que par bribes. C'est là une mutation décisive du regard sur les objets, que nous analysons également en observant les situations de fouille et $\mathrm{d}$ 'inventaire, en bonne méthode anthropologique pragmatique et réflexive.

L'utilisation de ce dépotoir centralisé témoigne de l'entrée véritable des établissements Paul Langeron dans l'industrie avec l'installation d'ateliers de moulage, alternative au façonnage manuel qui caractérisait l'entreprise depuis l'origine. C'est un virage vers la normalisation des produits et la massification de la fabrication. Plusieurs interprétations sont possibles du lien existant entre cette mutation technique et l'accroissement des déchets. L'adoption du moulage entraîne d'abord l'utilisation de nouveaux types de matériaux à usage limité et non réutilisable dans le cycle de production : les moules de plâtre qu'il faut évacuer sans pouvoir les utiliser comme remblai. Une telle évolution provoque également et inévitablement un accroissement des rebuts du fait des tâtonnements et expérimentations induits par toute innovation technique. Nous pouvons également supposer, sans pouvoir le vérifier, que les difficultés d'adaptation des ouvriers et de l'encadrement aux nouvelles techniques entraînent une augmentation du volume des rebuts. Il serait également intéressant d'évaluer le niveau d'exigence de la clientèle quant à la qualité des produits moulés (régularité des formes, des couleurs, des contenances), cette exigence étant une des causes de la mécanisation et de la normalisation morphologique des produits. La combinaison de ces facteurs a nécessité l'aménagement d'un dépotoir de masse, à l'accès stabilisé, et la rationalisation de l'élimination des déchets, par l'arasement des couches supérieures comme l'atteste la stratigraphie. La question des déchets industriels est devenue cruciale 
pour l'entreprise Langeron, comme elle l'est pour l'ensemble de la société de consommation : outre que celle-ci génère un volume de produits manufacturés et conséquemment de déchets toujours croissant, comme l'atteste d'ailleurs, en creux, la faible quantité de déchets domestiques retrouvés sur le dépotoir ${ }^{64}$, les évolutions techniques qu'elle induit ont également des retombées sur les déchets au niveau de la production. En l'occurrence, pour répondre à la demande de sa clientèle, la manufacture de céramique a dû adapter ses techniques de fabrication, ce qui a entraîné un accroissement des rebuts et débris non recyclables obligeant les industriels à centraliser et à rationaliser leur élimination. Ce phénomène nous est désormais mieux connu grâce aux deux campagnes de sondages archéologiques, qui ont également enrichi notre connaissance des productions Langeron et du quotidien de l'entreprise au $x x^{\mathrm{e}}$ siècle. Le déchet constitue en quelque sorte une articulation efficace entre histoire, archéologie et anthropologie, et cette méthode interdisciplinaire gagnerait à être appliquée pour d'autres sites repérés.

\section{Notes}

1. Qu'il me soit permis de remercier au passage pour leurs conseils Sophie de Beaune, Patrice Beck, Michel Maerten, Jean Rosen et Franck Faucher du service régional de l'archéologie de Bourgogne.

2. Alban Bensa, Après Lévi-Strauss. Pour une anthropologie à taille humaine, Paris, Textuel, 2010, p. 49.

3. Dictionnaire historique de la langue française, Paris, éditions Le Robert, 1992.

4. Florence Journot et Gilles Bellan, Archéologie de la France moderne et contemporaine, Paris, La Découverte, 2011, p. 97-113.
5. Frédéric Pillet, Le patrimoine minier $d u$ bassin de Blanzy, Montceau, Le Creusot, Dijon, éditions Faton, collection Itinéraires du Patrimoine $\mathrm{n}^{\circ} 214,1999$; Le patrimoine industriel métallurgique autour du Creusot, Dijon, éditions Faton, collection Itinéraires du Patrimoine $\mathrm{n}^{\circ}$ 243, 2001.

6. Michel MAerten, Les ateliers de potiers en Bourgogne du sud de l'antiquité au XIXe siècle, SaintVallier (71230), chez l'auteur, 2011.

7. Thierry Bonnot, L'industrie céramique des rives $d u$ canal $d u$ Centre, Saône-et-Loire, Paris, Éditions du patrimoine, collection Itinéraires du patrimoine $\mathrm{n}^{\circ} 151,1997$.

8. Victor Eugène Ardouin-Dumazet, Voyages en France (25 série). Basse-Bourgogne et Sénonais, Paris, Berger-Levrault, 1901, p. 31.

9. Digoin Céramiques et Sarreguemines Vaisselle (Digoin), Fauchon-Baudot (Paray-leMonial), Terres cuites de Bourgogne (Palinges) et Terreal (Chagny). Sarreguemines Vaisselle à Digoin, en redressement judiciaire courant 2015, a été rachetée en janvier 2016.

10. Sur les marques de fabrique ou certains documents du début du $x^{\mathrm{e}}$ siècle, le lieu-dit est rattaché à la commune voisine de Ciry-le-Noble.

11. «Cette maison, qui date de 1800, fabrique un très bon grès gris blanc, des cruchons, des flacons de formes diverses, destinés à contenir des liqueurs, des bouteilles à encre, des bocaux, des terrines », Georges VoGT, « Rapport de la classe 72. Céramique », Exposition universelle internationale de 1900 à Paris. Rapports du jury international, Paris, Imprimerie nationale, 1901, p. 79. Paul Langeron obtient la médaille d'argent.

12. AD Saône-et-Loire, cadastre napoléonien, Pouilloux, parcellaire section A2, L'essart.

13. AD Saône-et-Loire, état civil, actes de naissance, mariage, décès : 5E356/2,5E356/3 et 5E356/4, Pouilloux, et E dépôt 103 et 5E132/1, Ciry le Noble.

14. AD Saône-et-Loire, état civil, Pouilloux, cotes citées.

15. AD Saône-et-Loire, recensement de Pouilloux en 1836 et état civil, 5E356/5 et 5E356/6, Pouilloux.

16. Archives familiales Langeron, cahier manuscrit de Paul Langeron.

17. Archives de l'écomusée Creusot-Montceau, dépôt Langeron, lot A8 : société constituée devant notaire en 1898.

18. AD Saône-et-Loire, 6U 1042, tribunal de commerce de Charolles, faillites et liquidations judiciaires.

19. Archives de l'écomusée Creusot-Montceau, dépôt Langeron, lot A11 : accord Banque GarnierLangeron. 
20. Bulletin officiel de la propriété industrielle et commerciale, 1904, classe XIV, céramique et verrerie.

21. En octobre 1949, Octave-Lazare Langeron et son frère vendent leurs 175 parts de la société aux autres actionnaires ; Octave démissionne de sa fonction de gérant.

22. Archives familiales Langeron, acte de vente enregistré chez le notaire Paul Angellier à La Guiche.

23. Témoignage de $\mathrm{M}$. Henri Copin, de Pouilloux, employé par les Confiseries de Bourgogne de mai à août 1962. Archives familiales Langeron, extraits du greffe du tribunal de commerce de Charolles, papiers divers de la société Sacébon.

24. AM Pouilloux, matrices cadastrales.

25. En marche arrière pour le camion, selon le témoignage de M. Daniel Langeron, l'un des fils du dernier directeur de l'entreprise qui a passé son enfance sur place, jusqu'à la fermeture.

26. En reportant sur le plan cadastral certains repères de terrain, à partir des relevés effectués par un géomètre, nous pouvons préciser cette évaluation; le calcul donne $1084,37 \mathrm{~m}^{2}$, mais cette précision chiffrée est soumise à trop d'aléas pour être valable.

27. Si l'on admet une épaisseur moyenne des dépôts autour de 3,50 m. sur une surface de $25 \mathrm{~m}$. de large et $40 \mathrm{~m}$. de long, on parvient à un chiffre de 3500 mètres cubes.

28. Il peut s'agir d'une bouteille qui bave, c'està-dire qui n'est pas totalement imperméable, ou d'un produit sur lequel on distingue des bavures d'émail. " Monsieur Antoine » est vraisemblablement le patron des encres Antoine à Paris, important client des poteries de grès du Charolais.

29. Archives personnelles de Jacques Gaudiau.

30. C'est le cas à proximité immédiate du site industriel, dans la rivière Bourbince.

31. Sabine BARles, André GuIllerme et Laurence LESTEL, « Pollutions industrielle et réglementation des manufactures et ateliers en France au XIX ${ }^{\mathrm{e}}$ siècle : les textes fondateurs ", Documents pour l'histoire des techniques, $\mathrm{n}^{\circ} 17,2009$, p. 174-208.

32. Geneviève Massard-Guilbaud, Histoire de la pollution industrielle. France, 1789-1914, Paris, Éditions de l'EHESS, 2010.

33. Loi $n^{\circ} 75-633$ du 15 juillet 1975.

34. Ce mobilier intègrera les collections de l'écomusée Creusot-Montceau, labellisé Musée de France. L'écomusée a exposé une partie du mobilier dans le cadre d'une exposition en 2013, donnant lieu à la publication d'un catalogue : Thierry Bonnot et Jacques GAudiau, Trésors de dépotoir, Le Creusot, écomusée Creusot-Montceau, 2013.
35. Pour un inventaire complet, Thierry BonNot et Jacques GAUdiaU, « Dépotoir industriel des établissements de production céramique Paul-Langeron, le Pont-des-Vernes, Pouilloux ", rapport de sondage multigraphié, Service régional de l'archéologie de Bourgogne, arrêté de prescription $n^{\circ}$ 2012/105, Dijon, 2012. L'inventaire mobilier comporte 79 références pour le coulage, pour environ 40 modèles différents, 26 moules de calibrage, 18 pour l'estampage et 26 pour le pressage.

36. Archives Daniel Langeron, comptes rendus manuscrits des conseils d'administration de la SA des établissements Paul-Langeron, réunion du 25 mai 1919.

37. Archives de l'écomusée Creusot Montceau, dépôt Langeron, carton B1, bilans annuels et rapports au CA, 1919-1941.

38. William RATHJE et Cullen Murphy, Rubbish ! The archaeology of garbage, Tucson, University of Arizona Press, 2001 [1992], p. 34.

39. Sondage orienté nord-ouest/sud-est, d'une longueur de $17 \mathrm{~m}$. sur 3, $50 \mathrm{~m}$ de largeur et initialement 2, $30 \mathrm{~m}$ de profondeur.

40. Elle devient plus épaisse en direction du sud-est, c'est-à-dire vers la limite de parcelle. On passe d'une épaisseur de $30 \mathrm{~cm}$ à environ $60 \mathrm{~cm}$.

41. Pour la stratigraphie détaillée, Thierry Bonnot et Jacques GAUdiaU, « Dépotoir industriel des établissements Paul-Langeron.... ", second rapport de sondage, Service régional de l'archéologie de Bourgogne, arrêté n²014/175, Dijon, 2015.

42. F. Journot et G. Bellan, Archéologie de la France moderne et contemporaine, op. cit., p. 17.

43. Maurice Daumas, L'archéologie industrielle en France, Paris, Robert Laffont, 1980.

44. Jean-Claude BEAUNE, «Daumas (Maurice). L'archéologie industrielle en France », compte rendu in Milieux $\mathrm{n}^{\circ}$ 11-12, octobre 1982-janvier 1983, p. 112-113.

45. L'archéologie industrielle en France, revue créée en 1976 par le CILAC (Comité d'information et de liaison pour l'archéologie, l'étude et la mise en valeur du patrimoine industriel) fondé officiellement en 1978; [www.cilac.com].

46. Se reporter à la revue Milieux, publiée par l'écomusée entre 1980 et 1989 sous la direction de Jean-Claude Beaune, ainsi qu'au travail d'inventaire réalisé par Frédéric Pillet à la fin des années 1990, consultable sur la base Mérimée du ministère de la Culture.

47. F. Journot et G. Bellan, Archéologie de la France moderne et contemporaine, op. cit., p. 78-79.

48. Nous n'avons pas ici le temps de nous pencher sur les bornes de l'ère industrielle dans cette approche, autant discutées que la question de la 
qualification archéologique des recherches. Voir par exemple Bertrand GILLE, « L'archéologie industrielle », Milieux, ${ }^{\circ}$ 6, 1981, p. 22-23, ou Denis Woronoff, Histoire de l'industrie en France, du XVI siècle à nos jours, Paris, Le Seuil, 1994.

49. Philippe Boissinot, Qu'est-ce qu'un fait archéologique?, Paris, éditions de l'EHESS, 2015, p. 57.

50. Ibid.

51. Synonyme de généalogie, notamment chez Foucault, ibid., p. 11.

52. Ibid., p. 57-61. Philippe Boissinot vise aussi bien les fouilles des champs de bataille de la Grande Guerre que l'expérience du Déjeuner sous l'herbe de Jean-Paul Demoule (Jouy-en-Josas, 2010) ou l'exposition de Laurent Flutsch, Futur antérieur (Lausanne, 2002).

53. Ibid., p. 61.

54. Philippe Bruneau et Pierre-Yves Balut, "Positions ", RAMAGE, Revue d'archéologie moderne et d'archéologie générale, $\mathrm{n}^{\circ} 1,1982, \mathrm{p} .7$.

55. Ibid.

56. Ibid., p. 8.

57. Laurent Olivier, Le sombre abîme du temps, Paris, Le Seuil, 2008, p. 15.

58. Philippe Boissinot fait de ce point précis l'une des caractéristiques majeures de la discipline archéologique, définie comme un " noyau de pratiques autour de l'embrayage entre ces deux questions [...] : Qu'est-ce qu'il y a ici? Que s'est-il passé ici? ». Selon lui, « il ne se passe rien dans le volume ouvert par le chantier de fouilles mis à part le travail des archéologues, qui n'est évidemment pas l'objet de leurs propres recherches ». Pour l'anthropologue, le point de vue n'est pas le même. P. Boissinot, Qu'est-ce qu'un fait archéologique?, op. cit., p. 77-78.

59. Gilles BeLlan, "Archéologicité du présent ", NIKE (Nationale Informationsstelle für Kultutgüter-Erhaltung), n 6, 2011, p. 38-41.

60. Le Conseil national de la recherche archéologique a présenté, le 23 juin 2016, cette programmation, consultable en ligne : http: [//www. culturecommunication.gouv.fr/Thematiques / Archeologie/Etude-recherche/Programmationnationale]. Pour les périodes moderne et contemporaine, voir l'axe 14 .

61. Certaines bouteilles d'encre, par exemple, sont retrouvées pleines, encore cachetées.

62. Thierry BonNot, La vie des objets, d'ustensiles banals à objets de collection, Paris, éditions de la Maison des sciences de l'homme/Mission du patrimoine ethnologique, 2002.

63. Carlo Ginzburg, Mythes, emblèmes, traces. Morphologie et histoire, Paris, Verdier-Poche, nouvelle édition 2010 [1989], p. 247.

64. Le dépotoir a été alimenté avant les années 1960, donc avant la diffusion des produits plastiques et autres emballages, dans une période caractérisée par le recyclage, le remploi, la consigne des bouteilles de verre ou l'incinération. 